\title{
(W) Sofosbuvir plus ribavirin for treatment of hepatitis $C$ virus in patients co-infected with HIV (PHOTON-2): a multicentre, open-label, non-randomised, phase 3 study
}

\begin{abstract}
Jean-Michel Molina, Chloe Orkin, David M Iser, Francisco-Xavier Zamora, Mark Nelson, Christoph Stephan, Benedetta Massetto, Anuj Gaggar Liyun Ni, Evguenia Svarovskaia, Diana Brainard, G Mani Subramanian, John G McHutchison, Massimo Puoti, Jürgen K Rockstroh, for the PHOTON-2 study team*
\end{abstract}

\section{Summary}

Lancet 2015; 385: 1098-106 Published Online February 4, 2015 http://dx.doi.org/10.1016/ S0140-6736(14)62483-1 See Editorial page 1045 See Comment page 1052

*Members listed at end of paper

University of Paris Diderot, Paris 7 and Department of Infectious Diseases, Saint-Louis Hospital, Paris, France (ProfJ-M Molina MD); Barts Health National Health Service Trust, London, UK (C Orkin MD); Infectious Diseases Unit, Alfred

Hospital, Melbourne, VIC, Australia (D M Iser MBBS); HIV Unit, Internal Medicine Service, Hospital Universitario La Paz (IdiPAZ), Madrid, Spain (F-X Zamora MD); Chelsea and Westminster Hospital, St Stephens Centre, London, UK (M Nelson MBBS); Infectious Diseases Unit at Medical Department, Hospital of the Johann Wolfgang GoetheUniversity, Frankfurt, Germany (Prof C Stephan MD); Gilead Sciences, Foster City, CA, USA (B Massetto MD, A Gaggar MD, L Ni MA, E Svarovskaia PhD, D Brainard MD, G M Subramanian MD,

J G McHutchison MD); Division of Infectious Diseases, AO Ospedale Niguarda Ca' Granda, Milan, Italy (Prof M Puoti MD); Department of General Internal Medicine I, University Hospital of Bonn, Bonn, Germany (Prof J K Rockstroh MD) Correspondence to: Prof Jean-Michel Molina, Maladies Infectieuses et Tropicales, Hôpital Saint-Louis, Paris 75010, France jean-michel.molina@sls.aphp.fr

Background Although interferon-free regimens are approved for patients co-infected with HIV and genotype-2 or genotype-3 hepatitis $\mathrm{C}$ virus (HCV), interferon-based regimens are still an option for those co-infected with HIV and HCV genotypes 1 or 4 . These regimens are limited by clinically significant toxic effects and drug interactions with antiretroviral therapy. We aimed to assess the efficacy and safety of an interferon-free, all-oral regimen of sofosbuvir plus ribavirin in patients with HIV and HCV co-infection.

Methods We did this open-label, non-randomised, uncontrolled, phase 3 study at 45 sites in seven European countries and Australia. We enrolled patients (aged $\geq 18$ years) co-infected with stable HIV and chronic HCV genotypes 1-4, including those with compensated cirrhosis. Once-daily sofosbuvir (400 mg) plus twice-daily ribavirin (1000 mg in patients with bodyweights $<75 \mathrm{~kg}$ and $1200 \mathrm{mg}$ in those with weights $\geq 75 \mathrm{~kg}$ ) was given for 24 weeks to all patients except treatment-naive patients with genotype- $2 \mathrm{HCV}$, who received a 12 -week regimen. The primary efficacy endpoint was sustained virological response 12 weeks after treatment. We did analysis by modified intention to treat. This study is registered with ClinicalTrials.gov, number NCT01783678.

Findings Between Feb 7, 2013, and July 29, 2013, we enrolled 275 eligible patients, of whom 262 (95\%) completed treatment; 274 patients were included in the final analysis. Overall rates of sustained virological response 12 weeks after treatment were $85 \%$ (95\% CI 77-91) in patients with genotype-1 HCV, 88\% (69-98) in patients with genotype-2 HCV, 89\% (81-94) in patients with genotype-3 HCV, and 84\% (66-95) in patients with genotype-4 HCV. Response rates in treatment-naive patients with HCV genotypes 2 or 3 (89\% [95\% CI 67-99] and 91\% [81-97], respectively) were similar to those in treatment-experienced patients infected with those genotypes $(83 \%$ [36-100] and 86\% [73-94] respectively). There was no emergence of sofosbuvir-resistance mutations in patients with $\mathrm{HCV}$ viral relapse. Six (2\%) patients discontinued treatment because of adverse events. The most common adverse events were fatigue, insomnia asthenia, and headache. Four (1\%) patients had serious adverse events regarded as related to study treatment Additionally, four (1\%) patients receiving antiretroviral treatment had a transient HIV viral breakthrough; however none required changes in antiretroviral regimen.

Interpretation Sofosbuvir and ribavirin provided high rates of sustained virological response after 12 weeks of treatment in treatment-naive and treatment-experienced patients co-infected with HIV and HCV genotypes 1-4. The characteristics of this interferon-free combination regimen make sofosbuvir plus ribavirin a useful treatment option for this patient population.

Funding Gilead Sciences.

\section{Introduction}

In Europe, $25 \%$ of patients with HIV are co-infected with hepatitis $\mathrm{C}$ virus (HCV). ${ }^{1} \mathrm{HIV}$ exacerbates the morbidity of patients with HCV, even in those receiving highly active antiretroviral therapy. ${ }^{2-4}$ Patients co-infected with $\mathrm{HCV}$ and HIV have higher rates of liver failure and liver-related death than do those with HCV monoinfection..$^{2-4}$ As such, mortality in co-infected patients remains unchanged from before the introduction of combination antiretroviral therapy in 1996. ${ }^{5}$ Eradication of HCV in patients with $\mathrm{HCV}$ and HIV co-infection is associated with a reduction in liver-related events and in HIV progression and mortality not related to liver disease. ${ }^{6}$
Sofosbuvir is an oral nucleotide analogue inhibitor of the HCV non-structural 5B polymerase that has been approved for treatment of HCV genotypes 1-4. Sofosbuvir in combination with peginterferon and ribavirin is effective for treatment of HCV genotypes 1, 4, 5, and 6 , and sofosbuvir with ribavirin produces high rates of sustained virological response in patients with HCV genotypes 2 and 3..$^{-11}$ The PHOTON-1 study ${ }^{12}$ reported high rates (67-94\%) of sustained virological response after administration of sofosbuvir plus ribavirin to treatment-naive patients with HCV genotypes 1-3 and to treatment-experienced patients with HCV genotypes 2 and 3 co-infected with HIV and HCV. These findings have been used by the American 
Association for the Study of Liver Disease and the Infectious Diseases Society of America in jointly issued treatment guidelines. ${ }^{13}$ Ledipasvir in combination with sofosbuvir as a single-tablet regimen was approved in the USA for patients with HCV genotype 1 and in Europe for patients with genotypes 1 and 4, including for those with HIV co-infection. ${ }^{14}$ Other drugs in combination with sofosbuvir, such as daclatasvir and simeprevir, have been approved in some countries, although not specifically for use in patients with HIV and HCV co-infection.

We assessed the efficacy of sofosbuvir and ribavirin in patients co-infected with HCV and HIV. With this study, we aimed to expand on the results of PHOTON-1 by including patients with HCV genotype 4 , and to verify the benefit associated with a longer treatment duration for patients with HCV genotype 3 than for those with genotype- 2 infection, irrespective of previous treatment.

\section{Methods \\ Study design and patients}

We did this open-label, non-randomised, multicentre, uncontrolled trial at 45 clinical sites in Australia, France, Germany, Italy, Portugal, Spain, and the UK. Eligible patients were aged 18 years or older with a body-mass index of $18 \mathrm{~kg} / \mathrm{m}^{2}$ or more and chronic infection with HCV genotypes 1-4 (serum HCV RNA concentrations $\geq 10000 \mathrm{IU} / \mathrm{mL}$ ) and HIV-1. We included treatment-naive patients with HCV genotypes 1-4 and treatmentexperienced patients with HCV genotypes 2 or 3 (appendix). Roughly 20\% of patients could have compensated cirrhosis. Patients receiving antiretroviral therapy had to have CD4 T-cell counts of more than 200 cells per $\mathrm{mm}^{3}$, screening HIV-1 RNA concentrations of less than 50 copies per $\mathrm{mL}$, and to be on a stable antiretroviral regimen for more than 8 weeks before screening. Patients not receiving antiretroviral therapy for at least 8 weeks before screening (up to $10 \%$ of patients allowed) had to have CD4 T-cell counts of more than 500 cells per $\mathrm{mm}^{3}$. Antiretroviral regimens could contain combination emtricitabine and tenofovir plus ritonavir-boosted atazanavir or darunavir, efavirenz, raltegravir, or rilpivirine. Use of colony-stimulating drugs at screening or during the study was prohibited, as was active illicit drug use.

We did this study in accordance with the principles of the Declaration of Helsinki and Good Clinical Practice. All patients provided written informed consent.

\section{Procedures}

All patients received a once-daily oral dose of sofosbuvir $400 \mathrm{mg}$ in combination with twice-daily oral weight-based ribavirin $(1000 \mathrm{mg}$ in patients with bodyweights $<75 \mathrm{~kg}$ and $1200 \mathrm{mg}$ in those with weights $\geq 75 \mathrm{~kg}$ ). The treatment duration was 24 weeks for all patients except treatmentnaive patients with HCV genotype 2, who were treated for 12 weeks.
We identified compensated cirrhosis at screening with Fibroscan (Echosens, Paris, France) or FibroTest (BioPredictive, Paris, France), or by liver biopsy within 2 years of screening (appendix). We measured serum HCV RNA concentrations with Roche COBAS TaqMan HCV test (version 2.0) for use with the High Pure System (Roche Molecular Systems, Pleasanton, CA, USA; lower limit of quantitation [LLOQ] $25 \mathrm{IU} / \mathrm{mL}$ ). We defined HCV viral breakthrough as an HCV RNA concentration at or greater than the LLOQ after previous achievement of concentrations lower than the LLOQ while receiving treatment. We defined $\mathrm{HCV}$ viral relapse as an HCV RNA concentration at or greater than the LLOQ during the post-treatment period after achievement of concentrations lower than LLOQ at the end of treatment.

We did on-treatment assessments at 1-4 week intervals and included physical examination, review of drugs, and safety assessments (including assessments of renal function, haemoglobin concentrations, and liver function). Post-treatment assessments included safety and efficacy assessments 4 weeks and 12 weeks after the end of treatment. For analysis of viral resistance, we obtained blood samples before dosing (baseline) and at each subsequent visit. Any gene nucleotide changes in the non-structural 5B polymerase region, which might confer resistance to sofosbuvir, were assessed at baseline and at the time of HCV relapse or breakthrough with use of deep sequencing with a sensitivity of $1 \%$.

\section{Outcomes}

The primary efficacy endpoint was the proportion of patients with sustained virological response 12 weeks after treatment, defined as an HCV RNA concentration lower than the LLOQ 12 weeks after end of treatment.

\section{Statistical analysis}

We planned no formal comparisons between groups. Because there was no active control group, we did not do a sample-size calculation based on treatment difference, but rather established the expected $95 \%$ CI for point estimates of sustained virological response 12 weeks after treatment on the basis of historical response rates (appendix). We planned to enrol roughly 100 treatment-naive patients with HCV genotype 1, 50 treatment-naive patients with HCV genotypes 2 or 3, 20 treatment-naive patients with HCV genotype 4 , and 50 treatment-experienced patients with HCV genotypes 2 or 3 . We chose these target numbers on the basis of overall genotype prevalence in the HIV and $\mathrm{HCV}$ co-infected population, and to provide a reasonable estimate of sustained virological response 12 weeks after treatment, with appropriate $95 \%$ CIs. We included all patients who received at least one dose of study treatment.

We report data for the primary efficacy endpoint with point estimates and two-sided 95\% CIs (on the basis of the Clopper-Pearson method). We did exploratory multivariate logistic-regression analyses with forward stepwise selection 


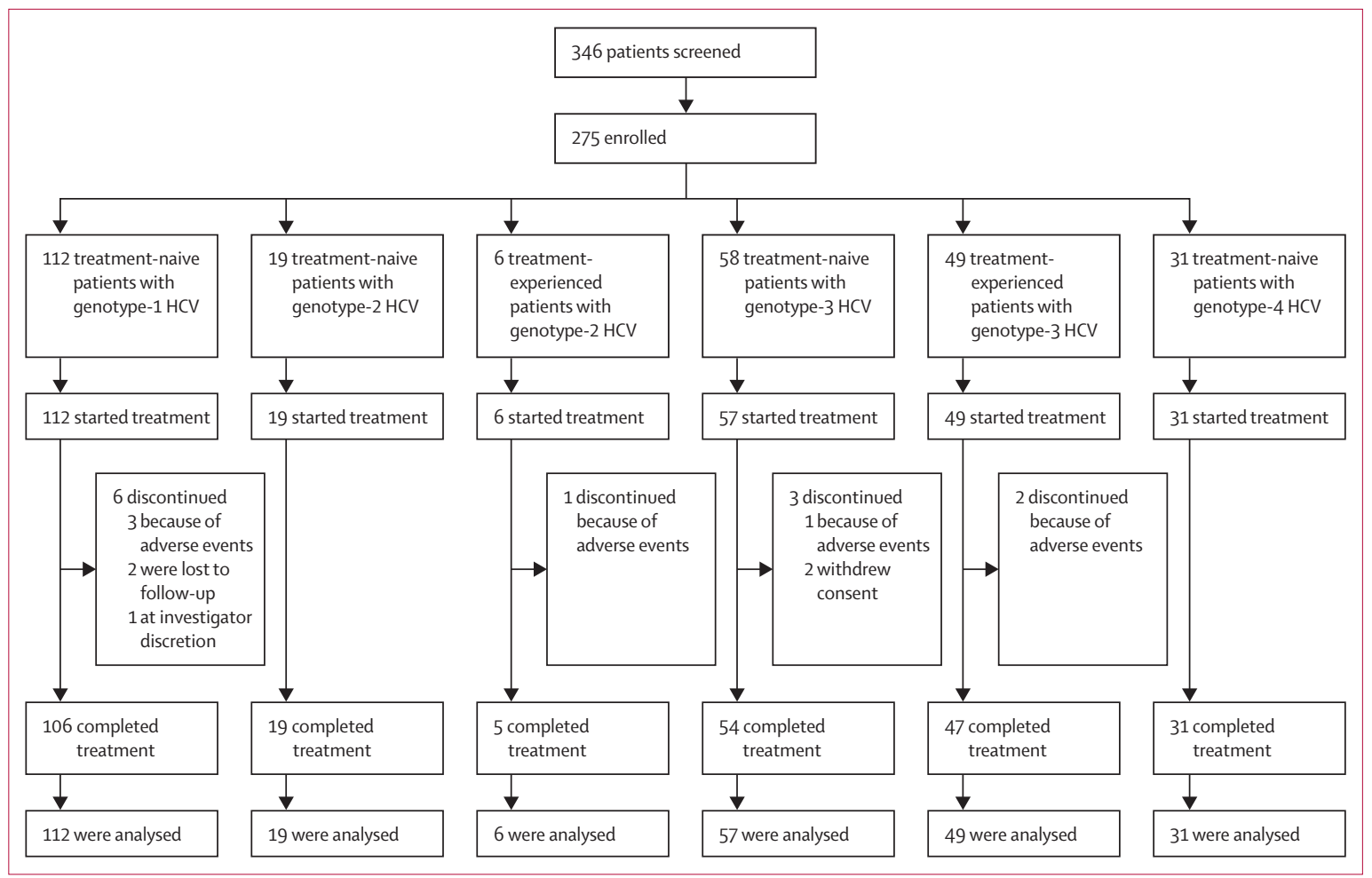

Figure 1: Study flowchart

$\mathrm{HCV}=$ hepatitis $C$ virus.

to identify independent predictors of sustained virological response. Analysis was by modified intention to treat. We did analysis with SAS (version 9.2). This study is registered with ClinicalTrials.gov, number NCT01783678.

\section{Role of the funding source}

The sponsor of the study designed and undertook the study, and collected and analysed the data, in collaboration with external investigators. The sponsor and JM-M interpreted data. The manuscript was drafted by employees of Niche Science and Technology (London, UK), who were paid by the sponsor. The authors had full access to all the data in the study and all authors participated in the decision to submit for publication.

\section{Results}

Figure 1 shows the study flowchart. Between Feb 7, 2013, and July 9, 2013, we enrolled 275 eligible patients, of whom 262 (95\%) completed treatment; 274 patients were included in the final analysis (figure 1, appendix). Table 1 shows demographic, disease, and baseline characteristics. Most patients were HCV treatment-naive (figure 1, table 1). Most of the treatment-experienced patients had genotype-3 HCV (figure 1, table 1). Overall, 54 (20\%) patients had cirrhosis, with a higher prevalence in treatment-experienced patients than in treatment-naive patients (table 1). Across treatment groups, $89-100 \%$ of patients were taking antiretroviral therapy (table 1 ).
Patients in all treatment groups had rapid and substantial decline in serum HCV RNA concentrations after start of treatment (table 2). By the second week of treatment, the proportion of patients with undetectable $\mathrm{HCV}$ ranged from $84 \%$ to $95 \%$ across all treatment groups except in treatment-experienced patients with genotype- $2 \mathrm{HCV}$ in whom the proportion was lower (table 2). By the fourth week of treatment, the proportion of patients with undetectable HCV was 96-100\% across all treatment groups (table 2). Rates of the primary endpoint of sustained virological response 12 weeks after treatment were $85 \%$ (95\% CI 77-91) in patients with genotype-1 HCV, 88\% (69-98) in patients with genotype-2 HCV, 89\% (81-94) in patients with genotype-3 HCV, and $84 \%$ (66-95\%) in patients with genotype-4 HCV (table 2). We noted similar response rates in patients with HCV genotypes 1a (84 [83\%] of 100 patients) and $1 \mathrm{~b}$ (ten [91\%] of 11 patients; figure 2). Of the 17 patients with HCV genotype 1 who did not achieve the primary endpoint, 14 (82\%) patients relapsed and three $(18 \%)$ patients were not assessable (two patients were lost to follow-up and one withdrew consent). For patients with HCV genotype 2, 17 (89\%) of the treatmentnaive patients and five $(83 \%)$ of the treatment-experienced achieved the primary endpoint (table 2). Two (67\%) of the three patients with HCV genotype 2 who did not achieve the primary endpoint relapsed (one treatment-naive and one treatment-experienced) and one could not be assessed (patient withdrew consent; table 2). 


\begin{tabular}{|c|c|c|c|c|c|c|}
\hline & \multirow{2}{*}{$\begin{array}{l}\text { Genotype } 1 \\
\text { Treatment-naive } \\
(n=112)\end{array}$} & \multicolumn{2}{|l|}{ Genotype 2} & \multicolumn{2}{|l|}{ Genotype 3} & \multirow{2}{*}{$\begin{array}{l}\text { Genotype } 4 \\
\begin{array}{l}\text { Treatment-naive } \\
(\mathrm{n}=31)\end{array}\end{array}$} \\
\hline & & $\begin{array}{l}\text { Treatment-naive } \\
\text { (12-week regimen; } \\
\mathrm{n}=19)^{*}\end{array}$ & $\begin{array}{l}\text { Treatment- } \\
\text { experienced }(n=6)\end{array}$ & $\begin{array}{l}\text { Treatment-naive } \\
(\mathrm{n}=57)\end{array}$ & $\begin{array}{l}\text { Treatment- } \\
\text { experienced }(n=49)\end{array}$ & \\
\hline Age (years) & $45(18-64)$ & $55(41-73)$ & $55(37-67)$ & $47(28-57)$ & $49(30-66)$ & $47(28-55)$ \\
\hline \multicolumn{7}{|l|}{ Sex } \\
\hline Male & $100(89 \%)$ & $15(79 \%)$ & $6(100 \%)$ & $38(67 \%)$ & $38(78 \%)$ & $24(77 \%)$ \\
\hline Female & $12(11 \%)$ & $4(21 \%)$ & 0 & $19(33 \%)$ & $11(22 \%)$ & $7(23 \%)$ \\
\hline $\operatorname{BMI}\left(\mathrm{kg} / \mathrm{m}^{2}\right)$ & $24 \cdot 5(17 \cdot 6-43 \cdot 6)$ & $22 \cdot 6(18 \cdot 3-26 \cdot 2)$ & $25 \cdot 4(21 \cdot 9-32 \cdot 2)$ & $24 \cdot 3(17 \cdot 5-38 \cdot 7)$ & $25 \cdot 0(19 \cdot 0-33 \cdot 2)$ & $23 \cdot 5(17 \cdot 3-33 \cdot 2)$ \\
\hline \multicolumn{7}{|l|}{ Race } \\
\hline White & $104(93 \%)$ & $18(95 \%)$ & $5(83 \%)$ & $54(95 \%)$ & $49(100 \%)$ & $29(97 \%)$ \\
\hline Black & $1(1 \%)$ & 0 & $1(17 \%)$ & 0 & 0 & $1(3 \%)$ \\
\hline Asian & $5(4 \% \%)$ & 0 & 0 & $2(4 \%)$ & 0 & 0 \\
\hline Other & $1(1 \%)$ & $1(5 \%)$ & 0 & $1(2 \%)$ & 0 & 0 \\
\hline Not reported & $1(1 \%)$ & 0 & 0 & 0 & 0 & $1(3 \%)$ \\
\hline \multicolumn{7}{|l|}{ IL28B genotype } \\
\hline CC & 48/111 (43\%) $\dagger$ & $12(63 \%)$ & $3(50 \%)$ & $30(53 \%)$ & $25(51 \%)$ & $9(29 \%)$ \\
\hline CT & $45 / 111(41 \%) \dagger$ & $5(26 \%)$ & $1(17 \%)$ & $21(37 \%)$ & $20(41 \%)$ & $14(45 \%)$ \\
\hline$\pi$ & $18 / 111(16 \%) \dagger$ & $2(11 \%)$ & $2(33 \%)$ & $6(11 \%)$ & $4(8 \%)$ & $8(26 \%)$ \\
\hline HCV RNA $\left(\log _{10} 1 \mathrm{U} / \mathrm{mL}\right)$ & $6 \cdot 3(0 \cdot 7)$ & $6.7(0.7)$ & $6.4(0.6)$ & $6 \cdot 3(0.7)$ & $6 \cdot 3(0.8)$ & $5.9(0.9)$ \\
\hline HCV RNA $\geq 6\left(\log _{10} I \mathrm{I} / \mathrm{mL}\right)$ & $79(71 \%)$ & $17(89 \%)$ & $5(83 \%)$ & $36(63 \%)$ & $37(76 \%)$ & $19(61 \%)$ \\
\hline Cirrhosis & $17(15 \%)$ & $1(5 \%)$ & $2(33 \%)$ & $3(5 \%)$ & $23(47 \%)$ & $8(26 \%)$ \\
\hline Interferon eligible & $88(79 \%)$ & $15(79 \%)$ & - & $44(77 \%)$ & - & $22(73 \%)$ \\
\hline CD4 cell count (cells per mm³) & $627(281)$ & $499(118)$ & $633(266)$ & $572(268)$ & $575(249)$ & $545(208)$ \\
\hline alanine aminotransferase $(\mathrm{U} / \mathrm{L})$ & $84(69)$ & $74(115)$ & $42(23)$ & $106(79)$ & $123(85)$ & $67(58)$ \\
\hline Estimated glomerular filtration rate $(\mathrm{mL} / \mathrm{min})$ & $110(27)$ & $95(23)$ & $106(13)$ & $101(25)$ & $105(27)$ & $96(22)$ \\
\hline \multicolumn{7}{|l|}{ Response to previous HCV treatment } \\
\hline Interferon intolerant & NA & NA & 0 & NA & $11(22 \%)$ & NA \\
\hline No response & NA & NA & $3(50 \%)$ & NA & $13(27 \%)$ & NA \\
\hline Relapse or breakthrough & NA & NA & $3(50 \%)$ & NA & $25(51 \%)$ & NA \\
\hline Receiving antiretroviral drugs & $109(97 \%)$ & $17(89 \%)$ & $6(100 \%)$ & $57(100 \%)$ & $46(94 \%)$ & $30(97)$ \\
\hline \multicolumn{7}{|c|}{ Tenofovir disoproxil fumarate and emtricitabine plus: } \\
\hline Efavirenz & $28 / 109(26 \%)$ & 6/17 (35\%) & $1 / 6(17 \%)$ & 9/57 (16\%) & $14 / 46(30 \%)$ & $7 / 30(23 \%)$ \\
\hline Raltegravir & $34 / 109(31 \%)$ & $1 / 17(6 \%)$ & $2 / 6(33 \%)$ & $8 / 57(14 \%)$ & $10 / 46(22 \%)$ & $6 / 30(20 \%)$ \\
\hline Darunavir ritonavir-boosted & $18 / 109(17 \%)$ & $5 / 17(29 \%)$ & $1 / 6(17 \%)$ & $18 / 57(32 \%)$ & $6 / 46(13 \%)$ & $8 / 30(27 \%)$ \\
\hline Atazanavir ritonavir-boosted & $15 / 109(14 \%)$ & $5 / 17(29 \%)$ & 0 & $12 / 57(21 \%)$ & $8 / 46(17 \%)$ & $4 / 30(14 \%)$ \\
\hline Rilpivirine & $5 / 109(5 \%)$ & 0 & 0 & $3 / 57(5 \%)$ & $2 / 46(4 \%)$ & $2 / 30(7 \%)$ \\
\hline Other & 9/109 (8\%) & 0 & $2 / 6(33 \%)$ & $7 / 57(12 \%)$ & $6 / 46(13 \%)$ & $3 / 30(10 \%)$ \\
\hline
\end{tabular}

For patients with HCV genotype 3, 52 (91\%) of the treatment-naive patients and $42(86 \%)$ of the treatmentexperienced patients achieved the primary endpoint (table 2). Of the 12 patients with HCV genotype 3 who did not achieve the primary endpoint, ten $(83 \%)$ relapsed (four were treatment-naive and six were treatment-experienced), one (8\%) was not assessable (patient withdrew consent), and one (8\%) had an on-treatment virological breakthrough (table 2). The patient who had the virological breakthrough was a treatment-experienced, 47-year-old man with cirrhosis and the CT genotype of the IL28B gene; plasma concentrations of GS-331007 and sofosbuvir suggest that the patient was adherent to study treatment. All five patients with HCV genotype 4 who did not achieve the primary endpoint had virological relapse (table 2). Across all treatment groups, rates of sustained virological response 12 weeks after treatment did not seem to be affected by the presence of cirrhosis, except in patients with genotype-1 HCV (appendix). For patients with other HCV genotypes, rates of sustained virological response did not differ significantly in those with and without cirrhosis (appendix).

Figure 2 and the appendix show results of the subgroup analysis of rates of sustained virological response 


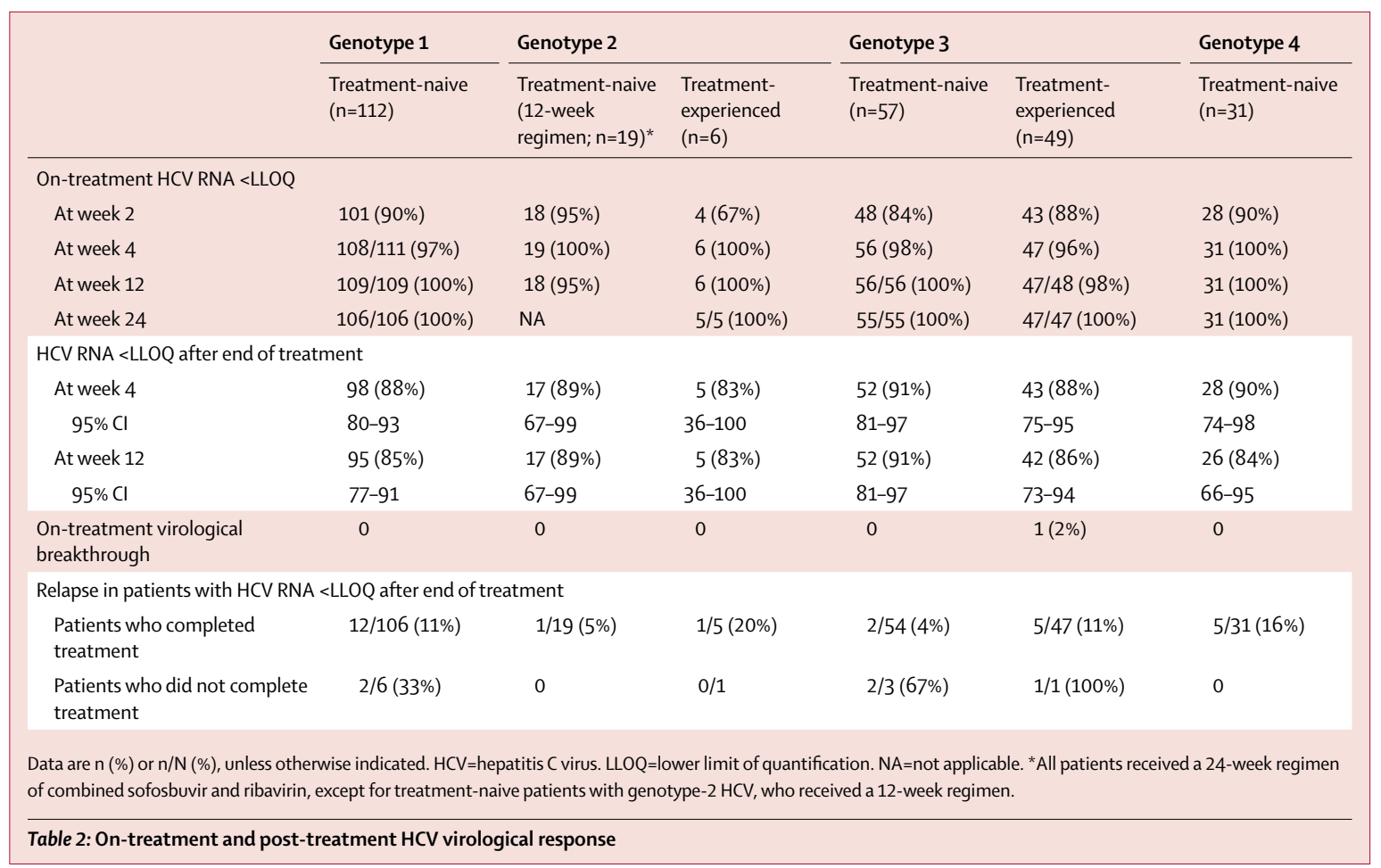

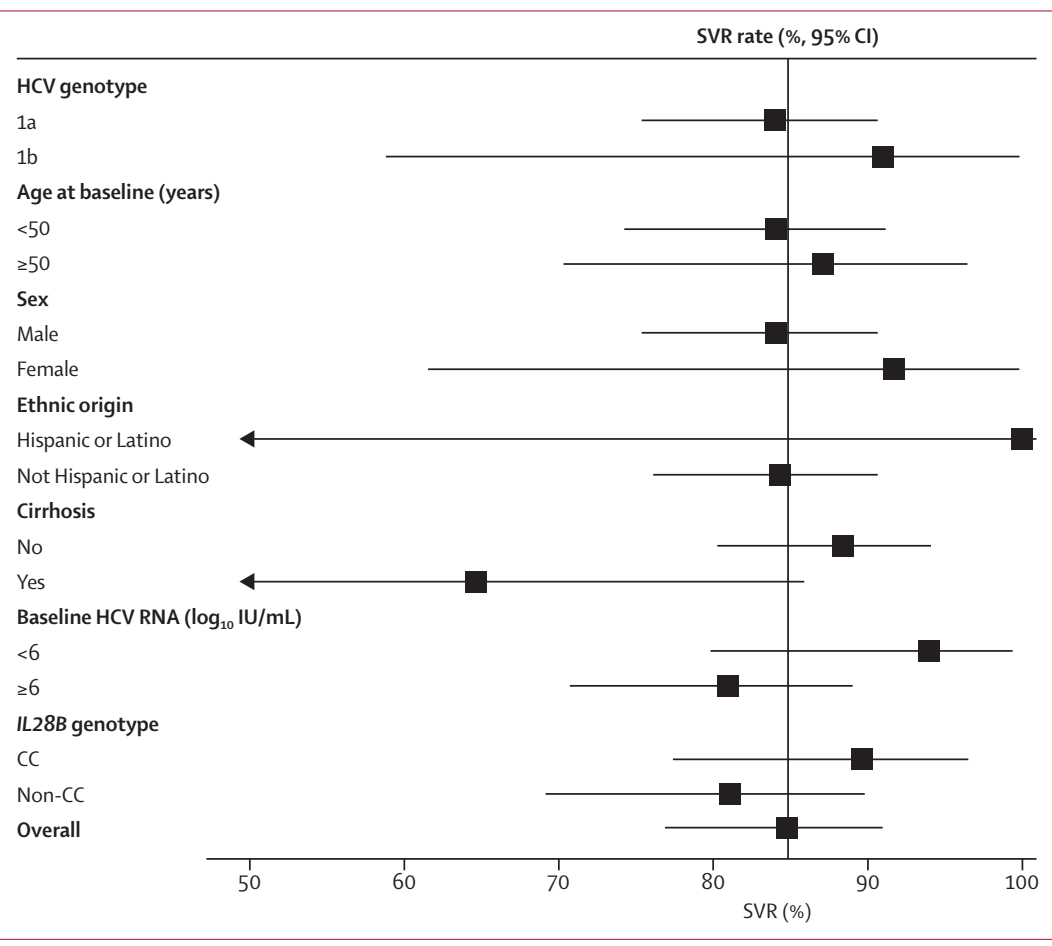

Figure 2: Forest plot for subgroup analysis of SVR rates 12 weeks after treatment

Rates are for treatment-naive patients with genotype- $1 \mathrm{HCV}$ who received sofosbuvir plus ribavirin for 24 weeks. $S V R=$ sustained virological response. $\mathrm{HCV}=$ hepatitis $C$ virus.

12 weeks after treatment in patients with HCV genotype 1 ; the appendix additionally shows subgroup results for patients with HCV genotypes 2-4. The exploratory multivariable analysis for baseline factors associated with achievement of the primary endpoint showed that in patients with genotype 1 , absence of cirrhosis (odds ratio 4.897, 95\% CI 1.422-16.858; $\mathrm{p}=0 \cdot 0118$; appendix) was predictive of sustained virological response 12 weeks after treatment. In patients with HCV genotypes $2-4$, no baseline factors were predictive of sustained virological response in exploratory multivariable analysis (appendix).

We undertook deep sequencing of the non-structural 5B polymerase region at baseline and virological failure in 31 patients who qualified for resistance testing. The $S 282 T$ variant, which is associated with resistance to sofosbuvir, was not present in any patient. We identified low levels of treatment-emergent variants in two patients with genotype-3a HCV: in one patient, who had on-treatment virological breakthrough, we identified the $L 159 F$ variant ( $2 \cdot 2 \%$ of the whole viral population), and in the other patient, who exhibited slow response to treatment, the L159F (5\%), S282N (3.4\%), and V321A (2.5\%) variants were also transiently detected. Additionally, two more patients-one with genotype-1 HCV and one with genotype-3a $\mathrm{HCV}-$ had the $L 159 \mathrm{~F}$ variant at time of relapse. $L 159 F$ and $V 321 A$ have only a slight effect on susceptibility to sofosbuvir in vitro, with a change of 1.3 times and 1.4 -times, respectively, in the median effective concentration. The S282N non-structural 5B polymerase variant did not replicate in vitro; therefore, we could not establish susceptibility to sofosbuvir.

Overall, 246 (90\%) patients reported at least one adverse event (table 3). Six (2\%) patients-all receiving the 24-week regimen-discontinued all study treatment 


\begin{tabular}{|c|c|c|c|c|c|}
\hline & $\begin{array}{l}\text { Genotype 2- } \\
\text { treatment-naive } \\
\text { (12-week } \\
\text { regimen; } n=19)\end{array}$ & $\begin{array}{l}\text { Genotypes } 1,3 \text {, } \\
\text { or } 4 \text {-treatment- } \\
\text { naive (24-week } \\
\text { regimen; } n=200)\end{array}$ & $\begin{array}{l}\text { Genotypes } 2 \text { or } \\
\text { 3-treatment- } \\
\text { experienced } \\
(24 \text {-week } \\
\text { regimen; } n=55)\end{array}$ & $\begin{array}{l}\text { Total }(24-\text { week } \\
\text { regimen; } \mathrm{N}=255)\end{array}$ & Total $(\mathrm{N}=274)$ \\
\hline Discontinuation of treatment because of an adverse event & 0 & $5(3 \%)$ & $1(2 \%)$ & $6(2 \%)$ & $6(2 \%)$ \\
\hline Serious adverse events & 0 & $10(5 \%)$ & $5(9 \%)$ & $15(6 \%)$ & $15(5 \%)$ \\
\hline Any adverse event & $17(89 \%)$ & $182(91 \%)$ & $47(85 \%)$ & $229(90 \%)$ & $246(90 \%)$ \\
\hline \multicolumn{6}{|l|}{ Common adverse events* } \\
\hline Fatigue & $5(26 \%)$ & $40(20 \%)$ & $11(20 \%)$ & $51(20 \%)$ & $56(20 \%)$ \\
\hline Insomnia & $3(16 \%)$ & $36(18 \%)$ & $5(9 \%)$ & $41(16 \%)$ & $44(16 \%)$ \\
\hline Asthenia & $1(5 \%)$ & $32(16 \%)$ & $11(20 \%)$ & $43(17 \%)$ & $44(16 \%)$ \\
\hline Headache & $1(5 \%)$ & $35(18 \%)$ & $7(13 \%)$ & $42(16 \%)$ & $43(16 \%)$ \\
\hline Nausea & $3(16 \%)$ & $32(16 \%)$ & $4(7 \%)$ & $36(14 \%)$ & $39(14 \%)$ \\
\hline Diarrhoea & $1(5 \%)$ & $24(12 \%)$ & $5(9 \%)$ & $29(11 \%)$ & $30(11 \%)$ \\
\hline \multicolumn{6}{|l|}{ Laboratory events } \\
\hline \multicolumn{6}{|l|}{ Decreased haemoglobin concentration (g/dL) } \\
\hline$<10$ & $1(5 \%)$ & $22(11 \%)$ & $3(5 \%)$ & $25(10 \%)$ & $26(9 \%)$ \\
\hline$<8.5$ & 0 & $1(<1 \%)$ & 0 & $1(<1 \%)$ & $1(<1 \%)$ \\
\hline Total bilirubin increased & $4(21 \%)$ & $32(16 \%)$ & $10(18 \%)$ & $42(16 \%)$ & $46(17 \%)$ \\
\hline Taking atazanavir & $4 / 4(100 \%)$ & $28 / 32(87 \cdot 5 \%)$ & $6 / 10(60 \%)$ & $34 / 42(81 \%)$ & $38 / 46(83 \%)$ \\
\hline Not taking atazanavir & 0 & $4 / 32(12 \cdot 5 \%)$ & $4 / 10(40 \%)$ & $8 / 42(19 \%)$ & $8 / 46(17 \%)$ \\
\hline \multicolumn{6}{|c|}{ Data are $n(\%)$ or $n / \mathrm{N}(\%)$. *Adverse events occurring in at least $10 \%$ of patients overall. } \\
\hline
\end{tabular}

because of an adverse event and 15 (5\%) had a serious adverse event (table 3, appendix). Four (1\%) patients had serious adverse events regarded as related to study treatment (two patients had anaemia, one patient developed thrombocytopenia and petechiae, and one patient had mania); all these events resolved by the end of follow-up. No patients died during the study.

The incidence of adverse events was similar between groups (table 3). The most common events were fatigue, insomnia, asthenia, and headache (table 3). Most adverse events were mild or moderate in severity. The most common laboratory abnormalities were decreased haemoglobin concentration and increased total bilirubin (appendix). Among patients who completed treatment, $30(11 \%)$ needed reductions in ribavirin dose. Of note, rates of sustained virological response 12 weeks after treatment in patients who needed ribavirin dose reduction $(80-100 \%)$ were similar to those in patients who completed full-dose treatment (80-95\%; appendix). We recorded grade 3 or 4 increases in total bilirubin in $46(17 \%)$ patients (table 3), of whom 38 (83\%) were receiving atazanavir as part of their antiretroviral regimen; only one patient had to stop atazanavir because of increased bilirubin (appendix). Of the eight (17\%) patients not taking atazanavir (table 2) who had grade 3 increases in total bilirubin, seven (9\%) patients had grade 1 or 2 levels at baseline and four (50\%) patients, who were all cirrhotic, had elevated direct bilirubin values from baseline onwards.

No clinically significant changes in HIV-1 RNA concentrations were noted in patients not taking antiretroviral drugs at baseline (appendix). In patients taking antiretrovirals at baseline, four (2\%) of 248 patients in the 24-week groups had a HIV-1 viral breakthrough; all these patients resupressed HIV-1 RNA by follow-up week 24 without any change in their antiretroviral regimen (appendix). We recorded a median change in absolute CD4 T-cell count from baseline to end of treatment of -91 cells per $\mathrm{mm}^{3}$ (IQR -14 to -184 ) for patients receiving 24 weeks of treatment $(n=255)$ and of -39 cells per $\mathrm{mm}^{3}$ (-22 to -102$)$ for those receiving 12 weeks of treatment $(n=19)$, which coincided with a decrease in absolute lymphocyte counts and was not associated with progression of HIV disease. By contrast, CD4 T-cell percentage values remained largely unaffected (appendix). Absolute CD4 cell counts returned to baseline concentrations by week 12 after treatment (appendix).

\section{Discussion}

Patients in our study achieved high rates of sustained virological response 12 weeks after treatment. These findings show that an interferon-free regimen of sofosbuvir plus ribavirin for 12 or 24 weeks can be highly effective for treatment of treatment-naive or treatmentexperienced patients co-infected with HIV and HCV genotypes 1-4. Results from the PHOTON-2 study are in keeping with those from the PHOTON-1 study ${ }^{12}$ done in the USA (panel). In PHOTON-1, treatment-naive patients with HCV genotypes 2 or 3 received sofosbuvir plus ribavirin for 12 weeks, and treatment-naive patients with HCV genotype 1 and treatment-experienced patients with 
Panel: Research in context

\section{Systematic review}

We did not do a systematic review of the treatment of patients co-infected with HIV and HCV because at the time the original protocol was finalised in October, 2012, no interferon-free regimens were available for treatment of patients with HCV. 48 weeks of peginterferon plus ribavirin, which was for many years the only regimen approved for the treatment of patients with HIV and HCV co-infection, was associated with low effectiveness and several safety concerns. Addition of a protease inhibitor to peginterferon plus ribavirin offered improved rates of efficacy, but significantly exacerbated safety issues and proved complicated to administer because of interactions with antiretroviral drugs used to treat HIV. To date, the PHOTON- 1 tria ${ }^{12}$ is the only other peer-reviewed report assessing an interferon-free regimen for treatment of patients co-infected with HIV and HCV.

\section{Interpretation}

Our findings provide the first clinical evidence that an interferon-free regimen can be highly effective for treatment of HCV genotype 4 in patients co-infected with HIV. Moreover, our results confirm the findings of PHOTON-1namely that sofosbuvir and ribavirin provide high rates of sustained virological response in patients with HCV genotypes 1-3 who are co-infected with HIV. In the near future, combinations that include more than one directacting antiretroviral drug are expected to enable shorter regimens, thus reducing treatment costs. However, characteristics of the sofosbuvir plus ribavirin combination, especially the scarcity of clinically significant interactions with antiretroviral drugs used in the treatment of HIV, will probably make this combination a useful treatment option for patients with HIV and HCV co-infection.

HCV genotypes 2 or 3 received sofosbuvir plus ribavirin for 24 weeks. Rates of sustained virological response 12 weeks after treatment were $76 \%$ in treatment-naive patients with HCV genotype 1, $88 \%$ for those with HCV genotype 2 , and $67 \%$ for those with HCV genotype 3 , and for treatment-experienced patients were $92 \%$ for patients with HCV genotype 2 and $94 \%$ for those with HCV genotype $3 .^{12}$ The notably higher response rate achieved by treatment-experienced patients receiving 24 weeks of treatment than by treatment-naive patients receiving 12 weeks of treatment led to the decision in PHOTON-2 to treat all patients with genotype- $3 \mathrm{HCV}$ for 24 weeks.

In the present study, the rate of sustained virological response $(85 \%)$ in treatment-naive patients with genotype-1 HCV was higher than that reported in the PHOTON-1 study (76\%) and in a previously reported phase 2 study in patients with HCV monoinfection who received the same regimen $(68 \%) \cdot{ }^{14}$ Of note, in the present study, the response rate 12 weeks after treatment in patients with genotype-1b HCV (91\%) was higher than that in those with genotype-1a infection $(84 \%)$, whereas the opposite was noted in the PHOTON-1 study, in which sustained virological response was achieved by $82 \%$ of patients with genotype-1a HCV and 54\% of those with genotype-1b infection. Reasons for these differences are unclear, but might indicate demographic differences between the populations or might simply be due to chance because these studies were not powered to identify differences between 12 -week rates of sustained virological response in patients with $\mathrm{HCV}$ genotypes $1 \mathrm{a}$ and $1 \mathrm{~b}$.

For patients with HCV genotype 2, the rates of sustained virological response were high, irrespective of previous treatment history or patient characteristics, although the small number of patients enrolled with genotype-2 HCV precludes definitive conclusions about the effect of these characteristics. For patients with HCV genotype 3, rates of sustained virological response were similar, irrespective of treatment history, interferon eligibility, and cirrhosis status. The response rates reported herein are within the range of those previously described for patients with HCV genotypes 2 or 3 alone to whom the same interferon-free combination of sofosbuvir plus ribavirin was given. ${ }^{9-11}$

The present study is the first report about use of sofosbuvir and ribavirin in patients with genotype-4 HCV co-infected with HIV. The high rate of sustained virological response achieved in this patient group in our study was close to that previously described in HCV monoinfected patients given a 12-week regimen of sofosbuvir plus peginterferon and ribavirin. ${ }^{9}$ Direct comparisons between these results and those of studies of sofosbuvir and ribavirin in HCV monoinfected patients are complicated by differences in baseline patient characteristics and comorbidities. In patients with genotype- 1 infection, assessment of 24 weeks of sofosbuvir and ribavirin in HCV-monoinfected patients (SPARE study) ${ }^{14}$ showed a lower rate of sustained virological response 12 weeks after treatment than that reported in PHOTON-2 (68\% vs 85\%); however, the number of patients in the SPARE study was small and included a disproportionate number of patients with various unfavourable characteristics. Findings from the VALENCE study and PHOTON-2, both done mainly in Europe, showed similar 12 -week response rates in patients with HCV genotype 2 given 12 weeks of sofosbuvir and ribavirin ( $97 \%$ and $89 \%$, respectively) and in those with genotype-3 infection given 24 weeks of sofosbuvir and ribavirin ( $85 \%$ and $89 \%$, respectively). The overall rate of adverse events in patients receiving 24 weeks of sofosbuvir and ribavirin in PHOTON-2 was lower than that in HCV-monoinfected patients in the VALENCE study. ${ }^{11}$ As was noted in HCV monoinfected patients who received sofosbuvir and ribavirin, patients with cirrhosis had numerically lower rates of sustained virological response after 12 weeks of treatment than did those without cirrhosis. The mechanism underlying historically lower rates of sustained virological response in patients with cirrhosis is not understood, but might be due to differences in the liver microenvironment, which could 
affect drug exposure, drug metabolism, or innate immune responses to $\mathrm{HCV}^{9,10}$

Altogether the results of our study provide strong evidence that HIV co-infection did not have a negative effect on the response to sofosbuvir therapy, as evidenced by similar rates of sustained virological response between patients with HCV and HIV co-infection and those with HCV monoinfection..$^{8-11}$ A previous study ${ }^{15}$ of patients with HCV and HIV co-infection showed no clinically significant drug interactions between sofosbuvir or any of its metabolites and several commonly used antiretroviral drugs, suggesting that sofosbuvir and ribavirin can be used without special dose adjustments in patients receiving most antiretroviral drugs.

Of note, no emerging S282T mutation associated with sofosbuvir resistance was noted in patients who relapsed after the end of treatment, suggesting that retreatment with sofosbuvir-based regimens, including sofosbuvir plus ledipasvir, might remain an option. ${ }^{16}$ We detected $L 159 F$ in five patients at failure. We identified low levels of emergent L159F mutation variants in four of five patients after treatment breakthrough or relapses. The clinical relevance of this mutation at such a low level is presently unclear, and this mutation was associated in vitro with a small decrease (less than two times) in sofosbuvir susceptibility. ${ }^{17}$

Reduction of CD4 cell counts is consistent with ribavirin-induced lymphopenia. Transient increases in total bilirubin were recorded mainly in patients receiving atazanavir and are consistent with ribavirin-induced haemolysis in the setting of atazanavir-mediated uridine glucuronosyltransferase $1 \mathrm{~A} 1$ inhibition, a finding that has been previously described. ${ }^{18-20}$ None of the patients with HIV viral breakthrough required changes to their antiretroviral regimen.

Limitations of the present study include the underrepresentation of women, black individuals, patients with cirrhosis, and those with advanced HIV disease. The fairly small size of some cohorts does not allow definitive conclusions to be made about the use of sofosbuvir in those groups. The low proportion of patients with HCV genotype 2 enrolled in this trial is reflective of the reported genotype distribution in co-infected patients in Europe. ${ }^{21}$ Notably, the preponderance of data in the scientific literature has shown a consistent and high response of patients with HCV genotype 2 to sofosbuvir and ribavirin, and our results are consistent with these historical data, even despite the small number of patients. The large number of patients enrolled with genotype- 3 HCV allowed for increased precision in the rates of sustained virological response 12 weeks after treatment in this group and in relevant subgroups for which data had previously been limited to one group of a phase 3 study. ${ }^{11}$ With development of new HCV regimens containing direct-acting antiretroviral drugs, the treatment landscape is evolving rapidly. However, few regimens have been assessed for treatment of patients with HCV genotypes 2 and 3 , and the absence of relevant drug interactions with antiretrovirals makes the regimen of sofosbuvir and ribavirin unique among interferon-free treatments.

Despite the limitations of this study, we feel that our findings provide important safety and efficacy data for the use of sofosbuvir and ribavirin across a broad population of co-infected patients with HCV genotypes 1-4. Further clinical studies are needed to confirm the efficacy and safety of sofosbuvir plus ribavirin in this difficult-to-treat patient population.

\section{Contributors}

$\mathrm{J}-\mathrm{MM}, \mathrm{BM}, \mathrm{JGMcH}$, and AG participated in the conception and design of the study, data acquisition, and data analysis and interpretation. $\mathrm{CO}$, DMI, F-XZ, MN, CS, MP, and JKR participated in data acquisition, and data analysis and interpretation. LN, ES, DB, and GMS contributed to data analysis and interpretation. All authors critically revised draft versions of the report and approved the final version for publication.

\section{PHOTON-2 study team members}

Laurent Cotte, Jacques Durant, Pierre-Marie Girard, Christine Katlama, Jean-Michel Molina, Yazden Yazdanpanah (France); Patrick Ingiliz, Hartwig Klinker, Thomas Lutz, Stefan Mauss, Jorg Petersen, Jürgen Rockstroh, Christoph Stephan (Germany); Andrea Antinori, Antonio Chirianni, Giovanni Di Perri, Adriano Lazzarin, Franco Maggiolo, Massimo Puoti, Giuliano Rizzardini (Italy); Juan Berenguer, Manuel Crespo, Juan Gonzalez-Garcia, Josep Mallolas, Juan Antonio Pineda, Carmen Quereda, Rafael Rubio (Spain); David Bell, Sanjay Bhagani, Graeme Cooke, Martin Fisher, Ranajababu Kulasegaram, Mark Nelson, Chloe Orkin (UK); David Baker Mark Bloch, David Iser, Gail Matthews, Joseph Sasadeusz (Australia)

\section{Declaration of interests}

J-MM has received honoraria for serving on advisory boards from Gilead, Janssen, Merck, ViiV and Bristol-Myers Squibb, and research grants from Merck and Gilead. MP has received personal fees for serving on temporary advisory boards or for participation in non-continuing medical education events, and has received research grants, from Abbvie, Astellas, Bristol-Myers Squibb, Boehringer Ingelheim, Beckman, Janssen, Gilead Sciences, GlaxoSmithKline, MSD, Novartis, Roche, Vertex, and ViiV. CO has received consulting fees or honoraria from Bristol-Myers Squibb, Gilead, Janssen, and MSD. MN has received grants, consulting fees, speakers fees, and support for travel from MSD, Janssen, Gilead, Abbott, Roche, Bristol-Myers Squibb, and ViiV. BM, AG, LN, ES, DB, GMS, and JGMcH are employees of Gilead Sciences. DMI, F-XZ, CS, and JKR declare no competing interests.

\section{Acknowledgments}

This study was sponsored by Gilead Sciences. We thank the staff and patients who participated in the study; Maryanne Lenoci and Sherri Paxton (Gilead Sciences) for clinical operations support; and Severina Moreira and Laila Guzadhur (Niche Science and Technology, London, UK) for writing and editorial support during development of this manuscript.

\section{References}

1 Rockstroh JK, Bhagani S. Managing HIV/hepatitis C co-infection in the era of direct acting antivirals. BMC Med 2013; 11: 234

2 Pineda JA, Romero-Gómez M, Díaz-García F, et al. HIV coinfection shortens the survival of patients with hepatitis $C$ virus-related decompensated cirrhosis. Hepatology 2005; 41: 779-89.

3 Pineda JA, García-García JA, Aguilar-Guisado M, et al. Clinical progression of hepatitis $\mathrm{C}$ virus-related chronic liver disease in human immunodeficiency virus-infected patients undergoing highly active antiretroviral therapy. Hepatology 2007; 46: 622-30.

4 Chen JY, Feeney ER, Chung RT. HCV and HIV co-infection: mechanisms and management. Nat Rev Gastroenterol Hepatol 2014; 11: $362-71$.

5 Ioannou GN, Bryson CL, Weiss NS, et al. The prevalence of cirrhosis and hepatocellular carcinoma in patients with HIV infection. Hepatology 2013; 58: 249-57.

6 Berenguer J, Rodríguez E, Miralles P, et al. Sustained virological response to interferon plus ribavirin reduces non-liver-related mortality in patients coinfected with HIV and Hepatitis C virus. Clin Infect Dis 2012; 55: 728-36. 
7 Gilead Sciences. Sovaldi (sofosbuvir) tablets: US prescribing information. December, 2013. http://www.gilead.com/ /media/ Files/pdfs/medicines/liver-disease/sovaldi/sovaldi_pi.pdf (accessed Oct 8, 2014).

8 Kowdley KV, Lawitz E, Crespo I, et al. Sofosbuvir with pegylated interferon alfa-2a and ribavirin for treatment-naive patients with hepatitis C genotype-1 infection (ATOMIC): an open-label, randomised, multicentre phase 2 trial. Lancet 2013; 381: 2100-07.

9 Lawitz E, Mangia A, Wyles D, et al. Sofosbuvir for previously untreated chronic hepatitis C infection. N Engl J Med 2013; 368: $1878-87$.

10 Jacobson IM, Gordon SC, Kowdley KV, et al. Sofosbuvir for hepatitis $C$ genotype 2 or 3 in patients without treatment options. N Engl J Med 2013; 368: 1867-77.

11 Zeuzem S, Dusheiko GM, Salupere R, et al. Sofosbuvir and ribavirin in HCV genotypes 2 and 3. N Engl J Med 2014; 370: 1993-2001.

12 Sulkowski MS, Naggie S, Lalezari J, et al. Sofosbuvir and ribavirin for hepatitis C in patients with HIV-1 coinfection. JAMA 2014; 312: 353-361.

13 American Association for the Study of Liver Diseases and the Infectious Diseases Society of America. Recommendations for testing, managing, and treating hepatitis C. www.hcvguidelines.org (accessed Oct 8, 2014).

14 Osinusi A, Meissner EG, Lee YJ, et al. Sofosbuvir and ribavirin for hepatitis $C$ genotype 1 in patients with unfavorable treatment characteristics: a randomized clinical trial. JAMA 2013; 310: 804-11.

15 Rodriguez-Torres M, Gaggar A, Shen G, et al. Sofosbuvir for chronic hepatitis $C$ virus infection genotype $1-4$ in patients co-infected with HIV. J Aquir Immune Defic Syndr 2015; published online Jan 23. DOI:10.1097/QAI.0000000000000516.
16 Osinusi A, Kohli A, Marti MM, et al. Re-treatment of chronic hepatitis $\mathrm{C}$ virus genotype 1 infection after relapse an open-label pilot study. Ann Intern Med 2014; 161: 634-38.

17 Svarovskaia ES, Dvory-Sobol H, Parkin N, et al. Infrequent development of resistance in genotype 1-6 hepatitis $C$ virus-infected subjects treated with sofosbuvir in phase 2 and 3 clinical trials. Clin Infect Dis 2014; 15: 1666-74.

18 Rodríguez-Nóvoa S, Morello J, González M, et al. Increase in serum bilirubin in HIV/hepatitis-C virus-coinfected patients on atazanavir therapy following initiation of pegylated-interferon and ribavirin. AIDS 2008; 22: 2535-37.

19 Zhang D, Chando TJ, Everett DW, Patten CJ, Dehal SS, Humphreys WG. In vitro inhibition of UDP glucuronosyltransferase by atazanavir and other HIV protease inhibitors and the relationship of this property to in vivo bilirubin glucuronidation. Drug Metab Dispos 2005; 33: 1729-39.

20 Sulkowski M, Pol S, Mallolas J, et al. Boceprevir versus placebo with pegylated interferon alfa-2b and ribavirin for treatment of hepatitis $\mathrm{C}$ virus genotype 1 in patients with HIV: a randomised, double-blind, controlled phase 2 trial. Lancet Infect Dis 2013; 13: 597-605.

21 Soriano V, Mocroft A, Rockstroh J, et al. Spontaneous viral clearance, viral load, and genotype distribution of hepatitis $\mathrm{C}$ virus (HCV) in HIV-infected patients with anti-HCV antibodies in Europe. J Hepatol 2008; 198: 1337-44. 